\title{
Identification and management of Lynch syndrome in the Middle East and North African countries: outcome of a survey in 12 countries
}

\author{
Mohammad Sina ${ }^{1,2} \cdot$ Zeinab Ghorbanoghli,4 $^{3}$ - Amal Abedrabbo ${ }^{5} \cdot$ Fahd Al-Mulla $^{6} \cdot$ Rihab Ben Sghaier $^{7}$. \\ Marie-Pierre Buisine $^{8} \cdot$ George Cortas $^{9} \cdot$ Ladan Goshayeshi $^{10} \cdot$ Andreas Hadjisavvas $^{11}$. Wail Hammoudeh ${ }^{12}$. \\ Waseem Hamoudi $^{13}$. Carol Jabari ${ }^{14,15} \cdot$ Maria A. Loizidou $^{11} \cdot$ Keivan Majidzadeh-A $^{1}$. Makia J. Marafie ${ }^{16}$. \\ Gurbankhan Muslumov ${ }^{17}$. Laila Rifai ${ }^{18}$. Rania Abu Seir ${ }^{19}$. Suzan M. Talaat ${ }^{20}$ - Berrin Tunca ${ }^{21}$. \\ Hadia Ziada-Bouchaar ${ }^{22}$ - Mary E. Velthuizen ${ }^{23}$. Ala I. Sharara ${ }^{24}$. Aysel Ahadova ${ }^{25,26}$. Demetra Georgiou ${ }^{27}$. \\ Hans F. A. Vasen ${ }^{3,4}$. on behalf of the Middle East Network on Hereditary Colorectal Cancer (HCCN-ME)
}

Received: 6 May 2020 / Accepted: 6 October 2020 / Published online: 24 October 2020

(c) The Author(s) 2020

\begin{abstract}
Background Lynch syndrome (LS), the most common inherited form of colorectal cancer (CRC), is responsible for 3\% of all cases of CRC. LS is caused by a mismatch repair gene defect and is characterized by a high risk for CRC, endometrial cancer and several other cancers. Identification of LS is of utmost importance because colonoscopic surveillance substantially improves a patient's prognosis. Recently, a network of physicians in Middle Eastern and North African (ME/NA) countries was established to improve the identification and management of LS families. The aim of the present survey was to evaluate current healthcare for families with LS in this region.

Methods A questionnaire was developed that addressed the following issues: availability of clinical management guidelines for LS; attention paid to family history of cancer; availability of genetic services for identification and diagnosis of LS; and assessment of knowledge of LS surveillance. Members of the network and authors of recent papers on LS from ME/NA and neighbouring countries were invited to participate in the survey and complete the online questionnaire.

Results A total of 55 individuals were invited and 19 respondents from twelve countries including Algeria, Azerbaijan, Cyprus, Egypt, Iran, Jordan, Kuwait, Lebanon, Morocco, Palestine, Tunisia, and Turkey completed the questionnaire. The results showed that family history of CRC is considered in less than half of the surveyed countries. Guidelines for the management of LS are available in three out of twelve countries. The identification and selection of families for genetic testing were based on clinical criteria (Amsterdam criteria II or Revised Bethesda criteria) in most countries, and only one country performed universal screening. In most of the surveyed countries genetic services were available in few hospitals or only in a research setting. However, surveillance of LS families was offered in the majority of countries and most frequently consisted of regular colonoscopy.

Conclusion The identification and management of LS in ME/NA countries are suboptimal and as a result most LS families in the region remain undetected. Future efforts should focus on increasing awareness of LS amongst both the general population and doctors, and on the improvement of the infrastructure in these countries.
\end{abstract}

Keywords Colorectal cancer $\cdot$ Lynch syndrome $\cdot$ Middle Eastern countries $\cdot$ North African countries

\section{Introduction}

Electronic supplementary material The online version of this article (https://doi.org/10.1007/s10689-020-00211-3) contains supplementary material, which is available to authorized users.

Zeinab Ghorbanoghli

z.ghorbanoghli@gmail.com

Extended author information available on the last page of the article
Colorectal cancer (CRC) is the third most commonly diagnosed cancer worldwide and the fourth leading cause of cancer-related death [1]. Although the incidence of CRC in Middle Eastern and North African (ME/NA) countries is low compared to Western countries [2], recent reports suggest that it is increasing rapidly [3]. 
One option for prevention of CRC is offering surveillance to individuals at high risk of inherited CRC. The most common hereditary form of CRC is Lynch syndrome (LS), which is responsible for 3\% of all cases of CRC [4]. LS is an autosomal dominant disorder caused by a pathogenic variant in one of the mismatch repair (MMR) genes, which include MLH1, MSH2 (EPCAM), MSH6 and PMS2. Loss of MMR function leads to the molecular phenotype of microsatellite instability (MSI) in tumours, which in turn drives carcinogenesis [4].

LS is characterized by a very high risk of developing CRC and endometrial cancer. The risk of developing a metachronous CRC is also high in Lynch syndrome individuals, therefore such patients should be offered a more extensive surgical treatment compared to sporadic CRC patients. LS families also show other cancers including cancers of the urinary tract, ovaries, stomach, pancreas, biliary tract, skin and cancers of the brain. Cancer risks and the spectrum of tumours depends on the type of underlying pathogenic variant [5]. The molecular phenotype of MSI characteristic for LS-associated tumours can be identified by PCR fragment length analysis of microsatellite markers or immunohistochemical (IHC) analysis of the MMR proteins. The diagnosis is confirmed by analysis of germline DNA.

Several studies have demonstrated that periodic examination of the colon leads to an improved prognosis and a substantial decrease in mortality [6, 7]. Identification of LS and the participation of families in surveillance programs is therefore of paramount importance.

In 2017, at a conference of the Palestinian Society of Gastroenterology in Jericho, a new network of doctors interested in hereditary CRC in ME/NA countries was established, now referred to as the Hereditary Colorectal Cancer NetworkMiddle East (HCCN-ME) [8]. The main goal of this network is to improve care for individuals with inherited CRC in these countries. In order to reach this goal, we first need a picture of current management of LS in the region. Thus, the aim of the present study was to survey the state of LS healthcare in ME/NA countries.

\section{Methods}

Our first task was to develop a questionnaire that addresses the most relevant issues (Supplementary file 1).The questionnaire contains three categories of questions: (1) general questions about the availability of guidelines for diagnosis and management of LS in each country, including questions concerning the attention paid by physicians to family histories of CRC (cancer), (2) questions about the genetic services available for the identification and diagnosis of LS, and (3) questions that assess general knowledge of the clinical management of LS patients.
All members of the network, and authors of recent articles on LS from ME/NA or neighbouring countries, were invited to participate in the survey. The invitation mail contained a link to the online questionnaire (developed using SurveyMonkey). The respondents came from 12 countries in the ME/NA region.

\section{Results}

A total of $22(40 \%)$ out of 55 professionals invited to participate in the survey responded. Three participants were excluded because they completed too few questions. The remaining 19 respondents were from twelve countries, including Algeria, Azerbaijan, Cyprus (two respondents), Egypt, Iran (two respondents), Jordan, Kuwait (two respondents), Lebanon, Morocco, Palestine (four respondents), Tunisia (two respondents), and Turkey (Table 1).

The respondents included eight clinical/molecular geneticists, four gastroenterologists, one surgeon, one researcher, one specialized nurse, one paediatrician, two biological scientists and one pathologist. Twelve of the 19 respondents were affiliated to a University Medical Centre.

The survey revealed that guidelines for the diagnosis and management of LS were available in three countries. In two countries, the respondents were unaware of clinical guidelines in their country. In five countries, most doctors pay appropriate attention to family history of CRC.

Genetic services were available in a research setting in one country, in only a few hospitals in six countries, in several hospitals in three countries and were absent in two countries. The most common explanation for the limited availability of these services was lack of funds in five countries, lack of interest or knowledge in four countries and a lack of trained geneticists in one country.
Table 1 Countries involved in the survey, including population (millions) (https://data.world bank.org/) 
Where genetic services were available, pedigree analysis was offered in ten countries, genetic counselling in eight countries, IHC or MSI-analysis in eight countries, MLH1promotor methylation or BRAF-analysis of tumours (performed to exclude methylation of the MLH1-promotor as cause of loss of MMR-function) in eight countries and (Sanger) sequencing or Next Generation Sequencing (NGS) in ten countries.

Clinical criteria were used for the identification of LS in eight of the 12 countries, while systematic screening of tumours was offered in only one country (i.e., Cyprus). Respondents from almost all countries (11 out of 12) stated that most LS families go unidentified in their country. By contrast, the respondent from Turkey reported that most LS patients in that country were identified.

With respect to the management of LS, colonoscopic surveillance is offered in 11 out of 12 countries and surveillance of the endometrium in five of the 12 countries. As regards the less common cancers observed in LS, surveillance of the urinary tract is offered in three countries and upper GI tract in five countries. Assessment of H.pylori infection was performed in four of the 12 countries. Extended surgery (subtotal colectomy) for CRC diagnosed at $<50-60$ years was an option in six of the 12 countries. A registry of families with Lynch syndrome was available in seven out of the 12 countries. The replies to five questions have not been shown because most respondents did not have information about the issue that was addressed. The outcome of the survey is summarized in Table 2 .

\section{Discussion}

Lynch syndrome is an example of an inherited form of cancer for which surveillance and early treatment is extremely effective [6, 7]. It is one of the most common inherited forms of cancers, affecting approximately $1 / 300$ people in Western countries [9]. Although data on the incidence of LS in Middle East and North African (ME/NA) countries are limited, studies suggest that LS accounts for a similar proportion of CRC compared to Western countries [10]. Based on these figures and an estimated population of 390 million in the 12 countries covered in the survey, we estimate that more than one million individuals in these countries have LS.
Table 2 Main outcomes from survey on current Lynch syndrome healthcare in the Middle East, North African and neighbouring countries

\begin{tabular}{lc}
\hline & Countries \\
& (total 12) \\
\hline 1. Guidelines available for management of LS? & 3 \\
2. Appropriate attention to family history of CRC by most doctors? & 5 \\
3. Genetic services available: & 3 \\
Several hospitals & 6 \\
Few hospitals & 1 \\
Only in research setting & 2 \\
Not available & \\
4. Explanations for limited genetic services? & 5 \\
Lack of finances & 4 \\
Lack of interest/knowledge & 1 \\
Lack of geneticists/genetic counsellors & \\
5. Strategies for identification of LS? & 8 \\
Clinical criteria, i.e., Amsterdam II criteria or Revised Bethesda guidelines & 1 \\
Universal screening of all new CRC or endometrial cancer & \\
6. Are most LS families identified? & 11 \\
Most not identified & 1 \\
Most identified & \\
7. Is surveillance offered? & 5 \\
Colonoscopic surveillance? & 5 \\
Endometrial surveillance? & 3 \\
Urinary tract? & 5 \\
Upper GI tract? & 5 \\
Helicobacter Pylori assessment? & \\
\hline Subtotal colectomy offered to patients with CRC <50 years & 5 \\
\hline
\end{tabular}


The current survey is the first study to provide information on prevailing LS healthcare in the ME/NA countries. The survey revealed that a family history of CRC receives appropriate attention in fewer than half the countries, and guidelines for the management of LS are available in only three of the 12 countries surveyed. The identification and selection of families for further genetic testing were generally based on clinical criteria, i.e., the Amsterdam criteria II or Revised Bethesda criteria, but universal screening was performed in one country. Genetic services are limited to only a few hospitals or a research setting in most countries. The surveillance offered in the majority of countries consists of regular colonoscopy, 1x/2 years, from age 20-25 years, while screening of the endometrium is available in less than half of the countries surveyed. In seven countries, a Hereditary Cancer Registry is available.

Ten years ago, a similar survey based on 30 respondents was performed in 14 European countries [11]. The results indicated that most countries devoted sufficient attention to a family history of CRC, although the quality of the obtained family history was considered suboptimal in some countries. Guidelines for the management of LS were available in most of the countries surveyed at that time. In addition, clinical criteria were mainly used for the selection of families for genetic testing (in all but one country), which is similar to the current survey. Nowadays, universal screening of all newly detected cases of CRC and endometrial cancer is recommended in the national guidelines of most European countries.

Another comprehensive survey of worldwide patterns of practice in the diagnosis and management of Lynch syndrome was performed recently by the International Mismatch Repair Consortium (IMRC)[12]. Data were collected from institutions in 21 countries (55 respondents) in Europe, North, Central and South America, Asia and Australasia. ME/NA countries were not included. Fifty-five percent of the respondents reported routine screening of newly identified LS-related cancers, and 27\% reported relying on clinical criteria together with selective tumour testing and germline analysis. Most institutions (64\%) also reported the use of multigene panels. Reported risk management practices included 1-2 yearly colonoscopy in almost all programs (98\%) and gynaecological screening in 78\%. Gastric cancer screening was recommended in 56\% of programs, especially so in Asia, North, Central and South America. The authors concluded that there is widespread heterogeneity in management practices for LS worldwide, which is probably due to the rapid pace of emerging technology and regional differences in resources.

A major strength of the survey is the large number of participating countries which taken together represent a population of 390 million people (Table 1). A concurrent limitation was the relatively low number of respondents from each country, which is probably attributable to a lack of awareness or interest in LS in these countries.

What are the implications of the results of this survey? In view of the finding that most families with LS in ME/ NA countries probably remain unidentified, the first and foremost question is how the recognition of these families might be improved and which tools should be used. In this respect, we must recognize that the economic outlook is poor in most of the included countries and therefore the best approach will likely vary between countries and depend on the available (financial) resources. Regardless of the economic situation, obtaining a detailed family history of (colorectal) cancer could be easily implemented in all countries. A detailed family history requires asking whether cancer occurs in first- and second-degree relatives, including the type of cancer and the age at diagnosis $[11,13]$. Online risk assessment tools can be used to assist with risk stratification and the management of patient and family screening [13]. In countries with limited financial resources, clinical criteria such as the revised Bethesda criteria can be used to select families for molecular testing of tumours, which is usually based on IHC analysis of the MMR proteins. In these countries, obtaining an appropriate family history is particularly important as it is essential to determine whether the clinical criteria have been met. In countries with sufficient financial resources, universal screening with IHC testing of all newly detected CRC and endometrial cancer below age 70 (or independent of age) is probably the best approach [14], although the cost-effectiveness of this approach in ME/NA countries remains to be determined.

For successful implementation of tumour analysis and subsequent germline analysis, a well-established infrastructure is needed. One of the reasons why genetic services are limited is that clinical geneticists or genetic counsellors are scarce in many countries. In view of the remarkable progress of genetic testing across a wide area of medicine, training of more geneticists and genetic counsellors should be promoted in all ME/NA countries and more attention should be paid to genetics in all medical curricula. In the interim, training of current healthcare professionals regarding hereditary cancer and genetic counselling should be organized, for example via e-learning modules [15]. In addition, an online forum could further assist the establishment of infrastructure through the use of shared resources as well as forming a supportive professional network to share experiences and opinions.

Registries of families with inherited cancer should also be established, with the aim of encouraging participation in prevention programs and to guarantee the continuity of lifetime surveillance programs [16].

Another key issue is the need to increase awareness of the importance of hereditary factors in the development of cancer amongst the general population as well as doctors. Information pamphlets for patients with (colorectal) cancer 
should contain a paragraph about hereditary cancer and the need for preventative measures in case of confirmed hereditary cancer. This information should also be provided on hospital websites accessible to the general public. To help improve knowledge about hereditary cancer among physicians in ME/NA countries, all major scientific conferences concerning CRC should include sessions on hereditary CRC, including polyposis and Lynch syndrome. In addition, guidelines should be developed that correspond to the specific situation in a country.

A further suggestion to help raise awareness of hereditary conditions among the public and healthcare professionals is the engagement of charities and non-profit governmental organisations to support and promote policy change. These organisations can also aid in the development of screening programmes, as well as universal genetic screening strategies for Lynch syndrome.

In conclusion, the current study shows that the identification and management of LS in ME/NA countries is still suboptimal. Future efforts should focus on increasing awareness of LS amongst both the general population and doctors, and on improvement of the infrastructure in these countries. A summary of our recommendations to improve LS care can be found in Table 3 .

\section{Compliance with ethical standards}

Conflict of interest The authors declare that they have no conflict of interest.

Open Access This article is licensed under a Creative Commons Attribution 4.0 International License, which permits use, sharing, adaptation, distribution and reproduction in any medium or format, as long as you give appropriate credit to the original author(s) and the source, provide a link to the Creative Commons licence, and indicate if changes were made. The images or other third party material in this article are included in the article's Creative Commons licence, unless indicated otherwise in a credit line to the material. If material is not included in the article's Creative Commons licence and your intended use is not permitted by statutory regulation or exceeds the permitted use, you will need to obtain permission directly from the copyright holder. To view a copy of this licence, visit http://creativecommons.org/licenses/by/4.0/.

\section{References}

1. Arnold M, Sierra MS, Laversanne M, Soerjomataram I, Jemal A, Bray F (2017) Global patterns and trends in colorectal cancer incidence and mortality. Gut 66(4):683-691

2. Center MM, Jemal A, Smith RA, Ward E (2009) Worldwide variations in colorectal cancer. CA Cancer J Clin 59(6):366-378

3. Dolatkhah R, Somi MH, Kermani IA, Ghojazadeh M, Jafarabadi MA, Farassati $F$ et al (2015) Increased colorectal cancer incidence in Iran: a systematic review and meta-analysis. BMC Public Health 15:997

4. Biller LH, Syngal S, Yurgelun MB (2019) Recent advances in Lynch syndrome. Fam Cancer 18(2):211-219 
5. Moller P, Seppala TT, Bernstein I, Holinski-Feder E, Sala P, Gareth Evans D et al (2018) Cancer risk and survival in path_MMR carriers by gene and gender up to 75 years of age: a report from the Prospective Lynch Syndrome Database. Gut 67(7):1306-1316

6. Jarvinen HJ, Aarnio M, Mustonen H, Aktan-Collan K, Aaltonen LA, Peltomaki P et al (2000) Controlled 15-year trial on screening for colorectal cancer in families with hereditary nonpolyposis colorectal cancer. Gastroenterology 118(5):829-834

7. de Jong AE, Hendriks YM, Kleibeuker JH, de Boer SY, Cats A, Griffioen G et al (2006) Decrease in mortality in Lynch syndrome families because of surveillance. Gastroenterology 130(3):665-671

8. Ghorbanoghli Z, Jabari C, Sweidan W, Hammoudeh W, Cortas G, Sharara AI et al (2018) A new hereditary colorectal cancer network in the Middle East and eastern mediterranean countries to improve care for high-risk families. Fam Cancer 17(2):209-212

9. Win AK, Jenkins MA, Dowty JG, Antoniou AC, Lee A, Giles GG et al (2017) Prevalence and penetrance of major genes and polygenes for colorectal cancer. Cancer Epidemiol Biomarkers Prev 26(3):404-412

10. Siraj AK, Prabhakaran S, Bavi P, Bu R, Beg S, Hazmi MA et al (2015) Prevalence of Lynch syndrome in a Middle Eastern population with colorectal cancer. Cancer 121(11):1762-1771

11. Vasen HF, Moslein G, Alonso A, Aretz S, Bernstein I, Bertario L et al (2010) Recommendations to improve identification of hereditary and familial colorectal cancer in Europe. Fam Cancer 9(2):109-115
12. Pan JY, Haile RW, Templeton A, Macrae F, Qin F, Sundaram $\mathrm{V}$ et al (2018) Worldwide practice patterns in lynch syndrome diagnosis and management, based on data from the international mismatch repair consortium. Clin Gastroenterol Hepatol 16(12):1901-1910 PMID: 29702294

13. Kallenberg FGJ, Aalfs CM, The FO, Wientjes CA, Depla AC, Mundt MW et al (2018) Evaluation of an online family history tool for identifying hereditary and familial colorectal cancer. Fam Cancer 17(3):371-380

14. Vasen HF, Blanco I, Aktan-Collan K, Gopie JP, Alonso A, Aretz $S$ et al (2013) Revised guidelines for the clinical management of Lynch syndrome (HNPCC): recommendations by a group of European experts. Gut 62(6):812-823

15. Douma KFL, Aalfs CM, Dekker E, Tanis PJ, Smets EM (2017) An e-learning module to improve nongenetic health professionals' assessment of colorectal cancer genetic risk: feasibility study. JMIR Med Educ 3(2):e24

16. Barrow P, Khan M, Lalloo F, Evans DG, Hill J (2013) Systematic review of the impact of registration and screening on colorectal cancer incidence and mortality in familial adenomatous polyposis and Lynch syndrome. Br J Surg 100(13):1719-1731

Publisher's Note Springer Nature remains neutral with regard to jurisdictional claims in published maps and institutional affiliations.

\section{Affiliations}

\section{Mohammad Sina ${ }^{1,2} \cdot$ Zeinab Ghorbanoghli,4 $^{3,0} \cdot$ Amal Abedrabbo $^{5} \cdot$ Fahd Al-Mulla $^{6} \cdot$ Rihab Ben Sghaier $^{7}$. Marie-Pierre Buisine ${ }^{8} \cdot$ George Cortas $^{9} \cdot$ Ladan Goshayeshi $^{10}$. Andreas Hadjisavvas ${ }^{11}$. Wail Hammoudeh ${ }^{12}$. Waseem Hamoudi ${ }^{13}$. Carol Jabari ${ }^{14,15}$. Maria A. Loizidou ${ }^{11} \cdot$ Keivan Majidzadeh-A $^{1}$. Makia J. Marafie ${ }^{16}$. Gurbankhan Muslumov ${ }^{17}$. Laila Rifai ${ }^{18}$. Rania Abu Seir ${ }^{19}$. Suzan M. Talaat ${ }^{20}$. Berrin Tunca ${ }^{21}$. Hadia Ziada-Bouchaar ${ }^{22}$. Mary E. Velthuizen ${ }^{23}$. Ala I. Sharara ${ }^{24}$. Aysel Ahadova ${ }^{25,26}$. Demetra Georgiou ${ }^{27}$. Hans F. A. Vasen ${ }^{3,4}$. on behalf of the Middle East Network on Hereditary Colorectal Cancer (HCCN-ME)}

1 Genetics Department, Breast Cancer Research Center, Motamed Cancer Institute, ACECR, Tehran, Iran

2 A. Nocivelli Institute for Molecular Medicine, Department of Molecular and Translational Medicine, University of Brescia, 25123 Brescia, Italy

3 Department of Gastroenterology and Hepatology, Leiden University Medical Center, Albinusdreef 2, 2333 ZA Leiden, The Netherlands

4 Dutch Hereditary Cancer Registry, Leiden, The Netherlands

5 Department of Pediatrics, Makassed Islamic Charitable Hospital, Jerusalem, Palestine

6 Department of Genetics and Bioinformatics, Dasman Diabetes Institute, P.O. Box 1180, 15462 Dasman, Kuwait

7 Cytogenetic, Molecular Genetics and Human Reproduction Biology - Farhat, HACHED Hospital, Sousse, Tunisia

8 Unit of Molecular Oncology and Genetics, Institute of Biochemistry and Molecular Biology, Lille University Hospital, Lille, France
9 Department of Gastroenterology, St. George Hospital Medical Center, University of Balamand Medical School, Beirut, Lebanon

10 Department of Gastroenterology and Hepatology, Faculty of Medicine, Mashhad University of Medical Sciences, Mashhad, Iran

11 Department of Electron Microscopy/Molecular Pathology, The Cyprus Institute of Neurology and Genetics, Nicosia, Cyprus

12 Department of Internal Medicine, Arabcare Hospital, Ramallah, Palestine

13 Department of Gastroenterology, The Royal Hospital, Amman, Jordan

14 Patient's Friends Society, Jerusalem, Palestine

15 Hebron University, Hebron, Palestine

16 Kuwait Medical Genetics Centre, Maternity Hospital, 13059 Safat, Kuwait

17 Colorectal Surgery Department, Scientific Center of Surgery, Baku, Azerbaijan 
18 Centre Hospitalier Universitaire IBN SINA, Rabat Instituts, Institut National D’ Oncologie Sidi Mohamed Ben Abdellah, BP 6213, Rabat, Maroc

19 Al-Quds University, Abu-Dis, Palestine

20 Ahmed Maher Teaching Hospital, Cairo, Egypt

21 Department of Medical Biology, Medical Faculty, Uludag University, Bursa, Turkey

22 Laboratory of Biology and Molecular Genetics, Faculty of Medicine, University 3, Rabah Bitat, Constantine, Algeria

23 Department of Genetics, University Medical Center Utrecht (Location WKZ), Utrecht, the Netherlands
24 Division of Gastroenterology, American University of Beirut Medical Centre, Beirut, Lebanon

25 Department of Applied Tumour Biology, Institute of Pathology, University Hospital Heidelberg, Heidelberg, Germany

26 Cooperation Unit Applied Tumour Biology, German Cancer Research Center (DKFZ), Heidelberg, Germany

27 Department of Clinical Genetics, London North West University Healthcare, London, UK 